\title{
Behavioural Modelling, Simulation, Test and Diagnosis of MEMS using ANNs
}

\author{
V. Litovski, M. Andrejević, \\ Electronics Faculty \\ University of Niš, \\ Niš, Serbia and Montenegro
}

\author{
M. Zwolinski \\ ECS \\ University of Southampton \\ Southampton, UK
}

\begin{abstract}
The design of Micro-Electrical-Mechanical Systems requires that the entire system can be modelled and simulated. Additionally, behaviour under fault conditions must be simulated to determine test and diagnosis strategies. While the electrical parts of a system can be modelled at transistor, gate or behavioural levels, the mechanical parts are conventionally modelled in terms of partial differential equations (PDEs). Mixed-signal electrical simulations are possible, using e.g. VHDL-AMS, but simulations that include PDEs are prohibitively expensive. Here, we show that complex PDEs can be replaced by black-box functional models and, importantly, such models can be characterized automatically and rapidly using artificial neural networks (ANNs). We demonstrate a significant increase in simulation speed and show that test and diagnosis strategies can be derived using such models.
\end{abstract}

\section{INTRODUCTION}

The International Technology Roadmap for Semiconductors (ITRS) [1] clearly identifies MEMS systems among the difficult challenges as feature sizes shrink below $100 \mathrm{~nm}$. Therefore there is a pressing need for innovative research in computer-aided-design methodologies and design tools for MEMS to tackle the challenges that will be faced by the semiconductor industry beyond the time horizon of the ITRS document.

When a system model is characterized, a design automation solution should allow a designer to extract, analyze, simulate, and optimize the system prior to the handoff to manufacturing. This provides confidence that the design will function as intended.

This comprehensive model requires a new infrastructure that supports the feedback loop between designer and manufacturer. This feedback loop should include a means of defining and relaying manufacturing constraints, verifying IC layouts, and demonstrating manufacturing-related issues to the designer.

Simulation and test signal synthesis are key issues in the development of such a model. Since the product development cycle in microsystem technology, or more specifically in Micro-Electro-Mechanical System (MEMS) technology, is rarely less than 5 years, design for manufacturability is becoming of prime importance. Inefficient design automation tools become a limiting factor for new applications of MEMS. Compared with digital, analogue or even mixed-signal products, poor tools may slow-down the product development cycle by orders of magnitude. Such delays are mainly created from a great number of trial-and-error steps caused by, among other things, immature simulation tools and inappropriate modelling methodologies.

This problem becomes even more drastic when testing and diagnosis are considered. Repetitive analyses are needed not only to verify and optimize the design but also for fault coverage verification and fault dictionary creation. We should not forget that we are speaking about systems on chip that encompass analogue and digital electronics and different (mostly mechanical) components described by specific models (most frequently by partial differential equations). An incomparably larger number of faults is expected in the electronic parts, meaning a vast number of simulations is expected. We cannot afford to simulate the mechanical parts at the lowest level. We need models of MEMS at a higher level in order to avoid the repetitive solution of systems of partial equations.

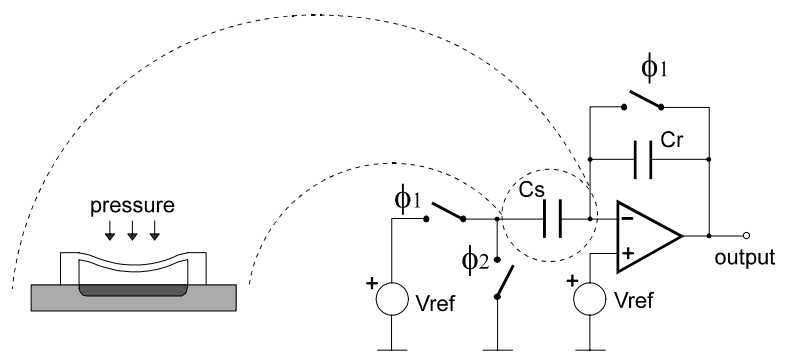

Figure 1. Micro-electro-mechanical capacitive pressure sensing system

In the next section we will discuss the standard approach to MEMS simulation. Then we will introduce black-box modelling by Artificial Neural Networks and apply this to a 
linear capacitor whose capacitance is controlled by pressure in a nonlinear manner. This model is then implemented in a behavioural system and circuit simulator to demonstrate the effectiveness of fault modeling and diagnosis.

\section{MEMS SIMULATION}

Consider the problem of simulating the simple circuit depicted in Fig. 1. This is a micro-electro-mechanical capacitive pressure sensing system. It consists of an electromechanical part that is a capacitor with a deflectable membrane such that a change in pressure results in a change in capacitance, and an electronic switched-capacitor network that generates a pulse train of fixed frequency. The pulse amplitude at the output is related to the capacitance value, so that the whole system converts pressure into a pulse amplitude.

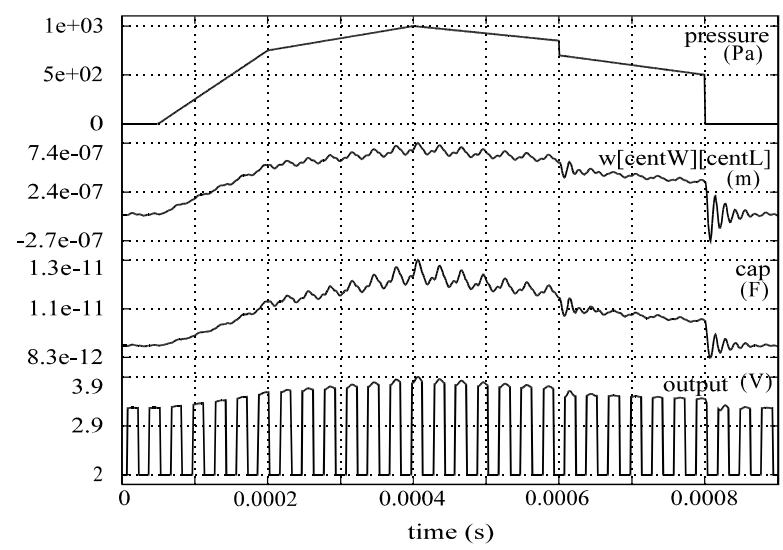

Figure 2. Time-domain simulation results for the system of Fig. 1. The displayed signals are pressure, displacement of the pressure sensor centre, sensor capacitance, and the output voltage.

Fig. 2 shows some simulation results of the system depicted in Fig. 1, using our simulator, Alecsis [2-4]. Alecsis is a general-purpose simulation framework, within which various simulation methods may be implemented. The mechanical and the electrical part are modelled within a single description and the simulation performs simultaneous evaluation of all system variables that are (in this case) displacements, voltages, currents, and states.

The concept proposed in this simulation system has been widely recognized [5-10]. In some cases this strategy has been adopted as a basis for further developments [11].

\section{BLACK-BOX MODELLING}

To start with, an ANN was created to characterize a lumped model of the capacitive transducer. The ANNs here are considered universal approximators [12], convenient for black-box device modelling. The process starts with extraction of the $C(p)$ dependence from the Alecsis simulation of the system in Fig. 1. Here $C$ stands for capacitance while $p$ denotes pressure. For this extraction the first and third traces of Fig. 2 were used to create Fig. 3.
The structure of the ANN used is a simple feed-forward network with only one hidden layer. The hidden neurons have sigmoidal activation functions, while the output neuron is linear. Table I contains the weights and thresholds of the ANN obtained after training with a standard algorithm, e.g. [13].

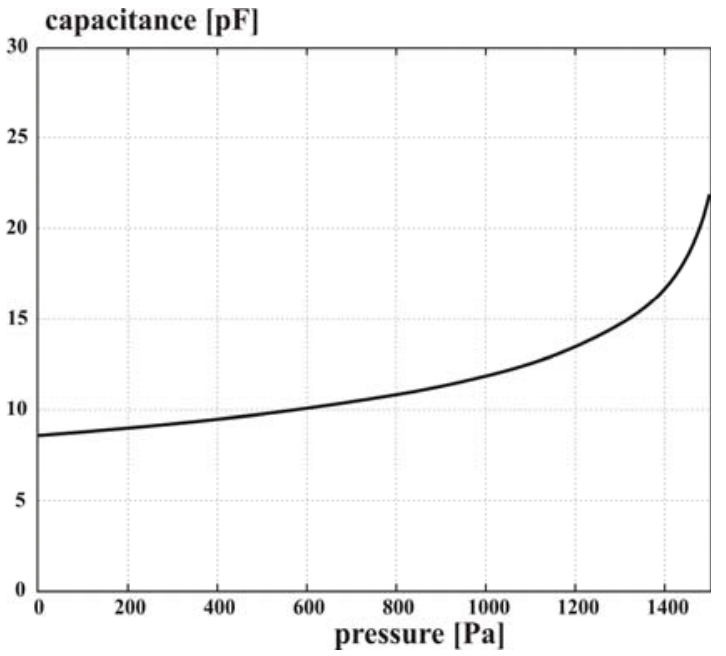

Figure 3. The capacitance of the membrane of Fig. 1 expressed as a function of pressure

TABLE I. WEIGHTS AND THRESHOLDS OF THE ANN USED TO APPROXIMATE THE CURVE OF FIG. 3

\begin{tabular}{|l|l|}
\hline \multicolumn{1}{|c|}{ Hidden layer neurons } & \multicolumn{1}{c|}{ Output layer neurons } \\
\hline $\mathrm{w}(1,1)(2,1)=33.1034$ & $\mathrm{w}(2,1)(3,1)=2.32691$ \\
$\mathrm{w}(1,1)(2,2)=3.92046$ & $\mathrm{w}(2,2)(3,1)=17.8609$ \\
$\mathrm{w}(1,1)(2,3)=4.04654$ & $\mathrm{w}(2,3)(3,1)=-15.9505$ \\
$\theta_{(2,1)}=-35.6658$ & $\theta_{(3,1)}=0.354662$ \\
$\theta_{(2,2)}=-3.88227$ & \\
$\theta_{(2,3)=-3.88324}$ & \\
\hline
\end{tabular}

These weights and thresholds are used as the parameters to a model of the ANN. In our case, this model is implemented in $\mathrm{C}++$ and linked directly into the Alecsis simulator, but it would be equally valid to write this model in VHDL(-AMS) or Verilog (-AMS). This ANN model is computationally much simpler and hence significantly more efficient than the partial differential equation model. Because the ANN is a universal model, one behavioural model is sufficient to cover a wide range of components.

The capacitor constitutive equation

$$
i_{c}=C(p) \frac{d v_{c}}{d t}
$$

was implemented according to [14].

The discretized and linearized model of the nonlinear capacitor may be expressed in a circuit form as depicted by 
Fig. 4. Note that, to apply this model we write code to calculate the response of the ANN that is $C(p)$, and its derivative with respect to $p$.

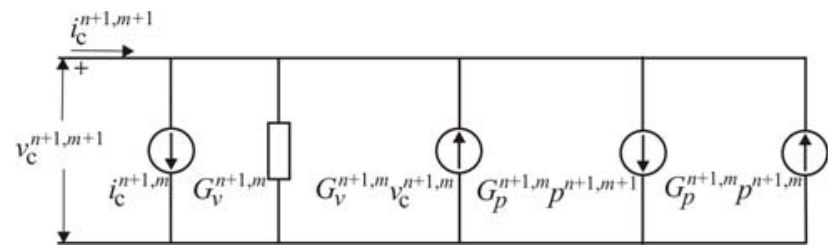

Figure 4. The linearized and discretized model of the capacitor controlled by pressure

\section{SimUlation RESUlTS}

The input (pressure) signal to the transducer used in the black-box model simulations is that shown in Fig. 2. A new simulation is performed now for the fault-free circuit (the same as Fig. 1) but with the PDE membrane model substituted by a lumped model of the capacitor expressed by the ANN. The simulation results, as shown in Fig. 5, are in excellent agreement with those obtained earlier, shown in the fourth trace of Fig. 3.

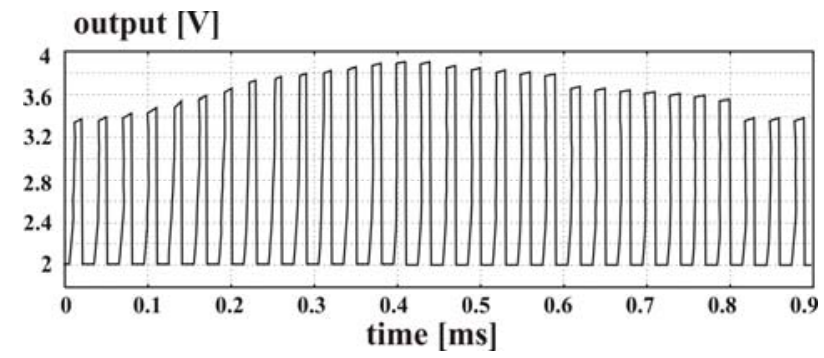

Figure 5. Simulation results of the circuit of Fig. 1 with the membrane substituted by a lumped capacitor

To emphasise the point, Fig. 5 was obtained using a model with only 5 network variables, compared with 1005 to describe the original distributed model. This enables inexpensive, repetitive simulation of the system when faults are inserted.

Simulation for testing and diagnosis will be considered next. For simplicity only five faults were selected as likely to occur:

1. S1-On: The feed-back switch of the operational amplifier stuck-at-closed

2. $\mathrm{Cr}+$ : The feed-back capacitor incremented by $+20 \%$

3. S1-Off: The feed-back switch of the operational amplifier stuck-at-open

4. Cr-: The feedback capacitor incremented by $-20 \%$

5. Cs-flat: The membrane stuck-at-flat (i.e. for some reason the membrane does not sense the pressure signal).

Once again, we use a parameterized ANN model to represent the membrane. For the first four faults, we use the fault-free model, but model fault conditions elsewhere in the system. Thus the lumped membrane model might be operating outside its normal range. In the fifth case, we model the faulty behaviour of the membrane by changing the parameters to the lumped, ANN model.

The simulation results for the fifth defect inserted in the circuit are given in Fig. 6. As a further simulation example, Fig. 7 shows the response of a faulty circuit with two faults present simultaneously: $\mathrm{Cr}+$ and S1-Off.

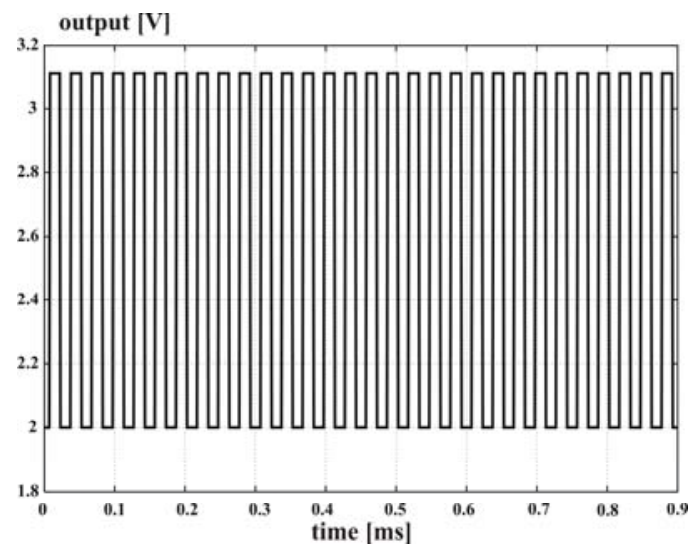

Figure 6. Response of the faulty circuit in the presence of the "membrane stuck-at-flat" fault

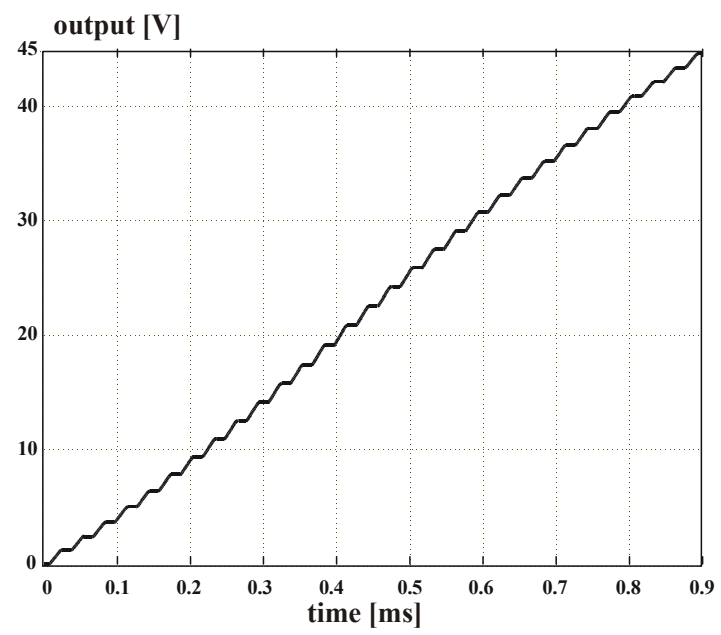

Figure 7. Response of the faulty circuit in the presence of two: $\mathrm{Cr}+$ and S1-Off faults

Note that, while fault model insertion in the electronic part (both digital and analogue) is well understood and easy to implement $[5,15]$, there is still much research to be done for mixed-signal electronic circuits and, unfortunately, even more work for non-electronic structures, the membrane being almost the simplest example.

Implementation of the above concept, however, allows for fast creation of a fault coverage list for a broad set of test signals and at the same time for creation of complex fault dictionaries needed to implement any diagnostics.

Table II contains the data that constitutes the fault dictionary for a single fault model. After the fault 
description, in the first column, the fault code is stated. There are five faults to be diagnosed. Accordingly, the fault dictionary has six rows enumerated from zero to five, the zeroth representing the fault-free (FF) system. These numbers are referred to as the fault codes. The last column in Table II shows the system response (DC voltage at the output, $\left.V_{\mathrm{oDC}}\right)$ for the fault-free and the faulty circuits.

TABLE II. FAULT DICTIONARY

\begin{tabular}{|lll|}
\hline \multicolumn{1}{r}{ Type } & \multicolumn{1}{r|}{ Code } & \multicolumn{1}{c|}{$\boldsymbol{V}_{\text {ODC }}[\mathbf{V}]$} \\
\hline $\mathrm{FF}$ & 000 & 2.829738 \\
$\mathrm{~S}_{1}-\mathrm{On}$ & 001 & 2.0 \\
$\mathrm{Cr}+$ & 010 & 2.691447 \\
$\mathrm{~S}_{1}-$ Off & 011 & 26.8489 \\
$\mathrm{Cr}-$ & 100 & 3.037174 \\
$\mathrm{C}_{\mathrm{s}}$ - flat & 101 & 2.555527 \\
\hline
\end{tabular}

This table was presented to an ANN learning algorithm and a new ANN was created that captures the mapping of Table II. Here, three linear output neurons are used in order to enable binary coding. Every output signal is then converted into binary digits as shown. Table III represents the data describing the new ANN used for diagnosis.

TABLE III. DIAGNOSIS WITH EXACT MEASUREMENT DATA

\begin{tabular}{|c|c|c|c|c|c|}
\hline Type & Code & $V_{\mathrm{o} \mathrm{DC}}[\mathrm{V}]$ & \multicolumn{3}{|c|}{ ANN output } \\
\hline \multirow{2}{*}{$\mathrm{FF}$} & \multirow{2}{*}{000} & \multirow{2}{*}{2.829738} & $1.5717 \mathrm{e}-05$ & -0.003626 & -0.0155174 \\
\hline & & & 0 & 0 & 0 \\
\hline \multirow{2}{*}{$\mathrm{S}_{1} \mathrm{ON}$} & \multirow{2}{*}{001} & \multirow{2}{*}{2.0} & $5.2172 \mathrm{e}-04$ & $5.24629 \mathrm{e} 04$ & 0.99966 \\
\hline & & & 0 & 0 & 1 \\
\hline \multirow{2}{*}{$\mathrm{Cr}+$} & \multirow{2}{*}{010} & \multirow{2}{*}{2.691447} & -0.0125319 & 0.991319 & 0.487591 \\
\hline & & & 0 & 1 & 0 \\
\hline \multirow{2}{*}{$\mathrm{S}_{1} \mathrm{OFF}$} & \multirow[b]{2}{*}{011} & \multirow{2}{*}{26.8489} & 0.0131203 & 1.00295 & 0.513954 \\
\hline & & & 0 & 1 & 1 \\
\hline \multirow{2}{*}{$\mathrm{Cr}-$} & \multirow{2}{*}{100} & \multirow{2}{*}{3.037174} & 0.999802 & 0.003041 & 0.00156264 \\
\hline & & & 1 & 0 & 0 \\
\hline \multirow[b]{2}{*}{$\mathrm{C}_{\mathrm{S}}$ flat } & \multirow{2}{*}{101} & \multirow{2}{*}{2.555527} & 0.999702 & -0.002840 & 1.01253 \\
\hline & & & 1 & 0 & 1 \\
\hline
\end{tabular}

After training, this ANN was used for diagnosis. We measure $V_{\mathrm{oDC}}$ at the output of the circuit and apply the measured value as an input to the ANN. The output of the ANN is the fault code of the respective fault as shown in the final column of Table III.

\section{CONCLUSIONS}

In order to decrease the time to market and increase the dependability of MEMS, tools are needed for the modelling, simulation and diagnosis of the entire system. To date this has only been possible by modelling the mechanical parts at the level of PDEs. This has led to excessive simulation times and has made fault simulation effectively impossible.
Here, we have demonstrated that accurate black-box modelling of the micro-mechanical parts is possible and, most significantly, that such models may be effectively characterized using ANNs.

Such an approach leads to rapid simulation and fault simulation and allows the further development of integrated design tools for entire Micro-Electro-Mechanical Systems.

\section{REFERENCES}

[1] International Technology Roadmap For Semiconductors 2003 Edition Modeling And Simulation http://public.itrs.net/Files /2003ITRS/Modeling2003.pdf

[2] Litovski, V., Maksimović, D., and Mrčarica, Ž., "Mixed-signal modeling with AleC++: Specific features of the HDL", Simulation Practice and Theory, Vol. 8, 2001, pp. 433-449.

[3] Mrčarica, Ž, Ilić, T., Glozić, D., Litovski, V., and Detter, H., "Mechatronic Simulation Using Alecsis: Anatomy of the Simulator", Proc. of Eurosim'95, Vienna, Austria, Sept. 1995, pp. 651-656.

[4] Mrčarica, Ž., Litovski, V., Detter, H., "Modeling And Simulation of Microsystems Using Hardware Description Language", Research Journal on Microsystem Technologies, Vol. 3, No. 2, Feb., 1997, pp. 80-85.

[5] Chappel, B.,"The fine art of IC design“, IEEE Spectrum, Vol. 36, No. 7, July 1999., pp. 30-34.

[6] Endemano, A., et al. "VHDL-AMS modeling and simulation of a planar electrostatic micromotor", J. of Micromechanics and Microengineering, Vol. 13, 2003, pp. 580-590.

[7] Gerlach, G., Schroth, A., and Klein, A., "Modellirung nichtelektrischer Komponenten in heterogenen und komplexen Mikrosystemen", Workshop: Methoden und Werkzeuge zum Entwurf von Microsystemen, Frankfut a.M., Jun 1996, pp. 12-21.

[8] Haase, J., Vermeiren, W., Clauss, C., und Schwarz, P.,"Erste Erfahrungen mit der simulation von Mixed-Signal-Schaltungen mit einem VHDL-AMS-Simulator", ASIM-Tagung, Simulationstechnik, Weimar, 17.09. 1999, Praxisforum, pp. 33-38.

[9] Haase, J., Schwarz, P., Trappe P., und Vermeiren, W. "Erfahrungen mit VHDL-AMS bei der Simulation heterogener Systeme", ITG/GI/GMM Workshop, "Methoden und beschreibungssprachen zur modellirung und verifikation von Schaltungen und Systemen", Frankfurt/M, 28-29.02.2000, pp. 167-175.

[10] Senturia, S. D., "CAD challanges for Microsensors, Microactuators, and Microsystems", Proceedings of the IEEE, Vol. 86, No. 8, August 1998., pp. 1611-1626.

[11] Zhang, T., Chakrabarty, K., and Fair, R. B., "Behavioral modeling and performance evaluation of microelectrofluidics-based PCR systems using SystemC", IEEE Trans. on CAD of Integrated circuits and Systems, Vol. 23, No. 6, June 2004, pp. 843-858.

[12] Scarselli, F., and Tsoi, A. C., "Universal approximation using feedforward neural networks: A survey of some existing methods and some new results", Neural Networks, Elsevier, Vol. 11, No. 1, 1998, pp. 15-37.

[13] Zografski, Z., "A novel machine learning algorithm and its use in modeling and simulation of dynamical systems", Proceedings of 5th Annual European Computer Conference, COMPEURO'91, Bologna, Italy 1991, pp. 860-864.

[14] Litovski, V., and Zwolinski, M., VLSI Circuit Simulation and Optimization, Chapman and Hall, London, 1997.

[15] Petković, P., Milovanović, D., Litovski, V., "Symbolic Fault Modeling And Test Generation of MOS Circuits", Microelectronics and Reliability, Vol. 37, No. 1, 1997, pp. 137-157. 